\title{
How sFlt-1 can help after preeclampsia diagnosis. (Mini-commentary on BJOG-20-0485.R2)
}

\author{
Daniel Rolnik ${ }^{1}$ and Aris Papageorghiou ${ }^{2}$ \\ ${ }^{1}$ Monash Health \\ ${ }^{2}$ University of Oxford
}

July 20, 2020

Measurement of maternal serum soluble fms-like tyrosine kinase 1 (sFlt-1) and placental growth factor $(\mathrm{PlGF})$, and the ratio between the two, has been shown to predict preeclampsia. In women with suspected preeclampsia, an sFlt-1: PlGF ratio below 38 rules out the need for delivery in the subsequent week with a negative predictive value of 99.3\% (95\% CI 97.9 to 99.9\%) (Zeisler H et al. , N Engl J Med 2016;374:13-22). This test may be of particular benefit for women at low risk of developing the disease in the short term, as it may reduce unnecessary follow-up, investigations and admissions (Cerdeira ASet al. , Hypertension 2019;74:983-990).

But do these biomarkers have a potential use in women after preeclampsia is diagnosed? This has not been studied extensively. In this issue of BJOG, Peguero and colleagues report the results of a study in which the changes in sFlt-1 and PlGF levels were examined in 63 women with early-onset preeclampsia from diagnosis to delivery (Peguero A et al. , BJOG 2020). Whilst no association between the change in PlGF levels and the development of adverse outcomes was evident, changes in sFlt-1 levels were significantly more pronounced in women who later developed complications and negatively associated with interval to delivery. This change (i.e., the delta sFlt-1) also outperformed a previously published risk score and the use of sFlt-1 at admission only.

Because prevention is better than cure, identifying high-risk women early and modifying their risk is desirable. Prediction of preeclampsia can now be achieved at 11 to 14 weeks of gestational age by calculation of individual patient risk. The risk calculation is based on a combined screening test that incorporates maternal characteristics, medical history and biomarkers (mean arterial pressure, uterine artery Doppler and PlGF) alongside first trimester combined screening for fetal aneuploidy. This test is particularly accurate for predicting early-onset preeclampsia, identifying nine out of ten of these severe cases (O'Gorman $\mathrm{N}$ et al. , Am J Obstet Gynecol. 2016;214(1):103 e1- e12). More importantly, when this high-risk group is given prophylaxis using aspirin $150 \mathrm{mg}$ from the first trimester to 36 weeks, the rate of preeclampsia before 37 and before 32 weeks is reduced by more than $60 \%$ and $90 \%$, respectively (Rolnik DL et al. , N Engl J Med. 2017;377(7):613-22). A recent implementation study demonstrated that early screening is not only feasible in a public health care setting, but is also associated with a significant reduction in the rate of preterm preeclampsia and nearly total physician compliance of aspirin use (29\% with usual care versus $99 \%$ when combined screening is used) (Guy GP et al. , BJOG 2020).

Nevertheless, not all women will undergo such early screening, and not all cases will be avoided by aspirin: some women will still develop preeclampsia, and early-onset disease will remain a significant cause of morbidity and mortality, disproportionally driving the disease burden. Hence, the findings of Peguero and colleagues are important and suggest that serial sFlt-1 measurements following a diagnosis of early-onset preeclampsia can still allow risk stratification - identifying women at lower risk of complications who may be eligible for expectant management, and those at higher risk who require prompt administration of steroids 
and timely transfer to tertiary health care facilities.

No disclosures: Completed disclosure of interest forms are available to view online as supporting information. 